\title{
Correction to: experimental investigations and soft computations for predicting the erosion mechanisms and peak outflow discharge caused by embankment dam breach
}

\author{
Kamran Kouzehgar $^{1}$ - Yousef Hassanzadeh ${ }^{1,2} \cdot$ Saeid Eslamian ${ }^{1,3} \cdot$ Mikaeil Yousefzadeh Fard $^{4}$. \\ Alireza Babaeian Amini ${ }^{5}$
}

Published online: 3 May 2021

(C) Saudi Society for Geosciences 2021

Correction to: Arabian Journal of Geosciences (2021) 14:616. https://doi.org/10.1007/s12517-021-06594-6

The original version of this paper was published with error. Authors' online corrections were submitted when journal production already in a state of performing corrections using the provided e-mail correction. Thus, all the latest corrections were not carried out.

The original article has been corrected.

The online version of the original article can be found at https://doi.org/ 10.1007/s12517-021-06594-6

Yousef Hassanzadeh

yhassanzadeh@tabrizu.ac.ir

1 Department of Civil Engineering, Najafabad Branch, Islamic Azad University, Najafabad, Iran

2 Department of Water Engineering, Center of Excellence in Hydroinformatics, Faculty of Civil Engineering, University of Tabriz, Tabriz, Iran

3 Department of Water Engineering, Isfahan University of Technology, Isfahan, Iran

4 Faculty of Civil Engineering, University of Tabriz, Tabriz, Iran

5 Faculty of Civil Engineering, University of Bonab, Bonab, Iran 